EGU2020-7938

https://doi.org/10.5194/egusphere-egu2020-7938

EGU General Assembly 2020

(c) Author(s) 2022. This work is distributed under

the Creative Commons Attribution 4.0 License.

\title{
NaFoLiCA: Synoptic-scale controls of fog and low cloud variability in the Namib Desert
}

\author{
Hendrik Andersen ${ }^{1,2}$, Jan Cermak ${ }^{1,2}$, Julia Fuchs ${ }^{1,2}$, Peter Knippertz ${ }^{1}$, Marco Gaetani ${ }^{3,4}$, Julian \\ Quinting ${ }^{1}$, Sebastian Sippel ${ }^{5}$, and Roland Vogt ${ }^{6}$ \\ ${ }^{1}$ Institute of Meteorology and Climate Research, Karlsruhe Institute of Technology, Karlsruhe, Germany \\ (hendrik.andersen@kit.edu) \\ ${ }^{2}$ Institute of Photogrammetry and Remote Sensing, Karlsruhe Institute of Technology, Karlsruhe, Germany \\ ${ }^{3}$ LISA/IPSL, CNRS, Université Paris Est Créteil, Université Paris, Créteil, France \\ ${ }^{4}$ LATMOS/IPSL, CNRS, Sorbonne Université, Université Paris-Saclay, Paris, France \\ ${ }^{5}$ Institute for Atmospheric and Climate Science, ETH Zurich, Zurich, Switzerland \\ ${ }^{6}$ Department of Environmental Sciences, University of Basel, Basel, Switzerland
}

This contribution presents new findings on synoptic-scale mechanisms that control the day-to-day variability of fog and low clouds (FLCs) in the Namib region.

FLCs are a defining element of the Namib-region climate and a crucial source of water for many species and ecosystems. Still, little is known on the processes driving Namib-region FLCs, in large part due to the very sparse observational records. Specifically, there is an ongoing debate in the scientific literature concerning the relevance of different mechanisms responsible for fog formation in the region. In this contribution, a new long-term satellite-based data set of FLC occurrence is used in conjunction with reanalysis data and backtrajectories to systematically analyze dynamical and thermodynamical differences between days with and without FLCs in the central Namib during two different seasons. The main findings are:

- Central-Namib FLCs are nearly always associated with the advection of marine-boundary-layer air masses.

- The variability of the overall FLC coverage in the central Namib is largely driven by dynamics at the synoptic scale.

- Seasonally different synoptic-scale mechanisms determine the probability of the occurrence of FLCs in the central Namib.

The findings lead to a better understanding of Namib-region FLCs and help broaden the understanding of low clouds along the southwestern African coastline and the southeast Atlantic. 